\title{
Growth delay due to insulin-like growth factor I resistance
}

INSERM

\section{Source}

INSERM. (1999). Orphanet: an online rare disease and orphan drug data base. Growth delay due to insulin-like growth factor / resistance. ORPHA:73273

Growth delay due to IGF-I resistance is characterised by variable intrauterine and postnatal growth retardation and elevated serum IGF-I levels. Addition features include variable degrees of intellectual deficit, microcephaly and dysmorphism (broad nasal bridge and tip, smooth philtrum, thin upper and everted lower lips, short fingers, clinodactyly, wide-set nipples and pectus excavatum). 